\title{
An Optical Scanner Based on Cantilever-type Electrostatic Zipping Actuators
}

\author{
Smitha M. N. Rao ${ }^{1}$, Praveen Pandojirao-Sunkojirao ${ }^{1}$, Naresh Dhaubanjar ${ }^{1}, \mathrm{Mu} \mathrm{Chiao}^{2}$ and \\ J.-C. Chiao ${ }^{1}$ \\ ${ }^{1}$ Department of Electrical Engineering, The University of Texas at Arlington, USA \\ ${ }^{2}$ Department of Mechanical Engineering, The University of British Columbia, Canada
}

\begin{abstract}
This paper discusses modeling, design, fabrication and characterization of an optical scanner based on cantilever-type electrostatic zipping actuators. The electrostatic actuator has been designed to achieve high displacements for large optical scanning angles at lower actuation voltages. The zipping actuators are fabricated using multi-layer polysilicon foundry fabrication processes. The electrostatic force between the cantilever and the bottom electrode on the substrate pulls the cantilever down. With a warped cantilever, the force closes the gap from the anchored end and gradually the zipping effect actuates the entire cantilever. In our design, mechanical structures are arranged to avoid electrical shortcircuit. With various annealing temperatures, the warped angles are controllable. The cantilever serves as a reflective surface and the high out-of-plane displacement is used to steer a reflected laser beam for imaging and scanning applications. In this paper we present the design considerations in electrostatic zipping actuator displacement and control as well as the arrangement for optical scanning.
\end{abstract}

Keywords: optical scanner, cantilever-type zipping actuator, electrostatic actuator

\section{INTRODUCTION}

Optical MEMS is a promising technology with applications in telecommunications, high-resolution displays, and medical imaging. Electrostatic actuators have the advantages of low power consumption, high actuation speeds, and fabrication compatibility [1]. Large deflection and force can be achieved by using the cantilever type electrostatic zipping actuator. Electrostatic actuators with an S-shaped film element and deformed membrane have been reported with high displacements $[2,3]$. Legtenberg et al. have demonstrated laterally compliant curved electrode zipping actuators [4]. Optical fibers have been aligned using curved electrodes [5]. The large gap between the top and the bottom electrodes increases the voltage required to actuate the actuator. The zipping actuator works well if the angle between both the electrodes is small at the beginning of zipping action [6]. The zipper actuator can be fabricated using various commercial fabrication processes such as SOI (silicon on insulator), bulk micromachining and surface micromachining. These batch fabrication techniques are monolithic and thus the fabrication cost could be relatively lower.

When the zipping actuator is actuated, the bottom fixed electrode pulls the top electrode down, reducing the gap between the electrodes. At a sufficiently high voltage, the top electrode snaps down on the bottom electrode thus shorting the circuit. In order to prevent this shorting, conventional designs include a thin layer of insulating material to electrically separate the two electrodes. A thin layer of silicon dioxide or silicon nitride is generally used. To circumvent the problem of electrical shorting and dielectric breakdown, a new design of a surface micromachined cantilever-type zipper actuator has been presented [7]. The actuator was fabricated using the multi-layer polysilicon foundry fabrication process PolyMUMPS. This actuator can be utilized in microwave and millimeterwave waveguide applications for switching, tuning and phase shifting and imaging applications. The analysis and experimental results demonstrated that the design can withstand high voltages without shorting and is capable of producing high deflections. In this paper, the design considerations involved in displacement of the zipper actuator are presented. The actuator is to be used for optical imaging and scanning applications. The optical arrangement for utilizing a gold coated reflective surface of the zipping actuator to steer a laser beam for imaging applications is then presented.

MEMS/MOEMS Technologies and Applications III, edited by Jung-Chih Chiao, Xuyuan Chen, Zhaoying Zhou, Xinxin Li,

Proc. of SPIE Vol. 6836, 683617, (2007) · 0277-786X/07/\$18 · doi: 10.1117/12.767801

Proc. of SPIE Vol. 6836 683617-1 


\section{ACTUATOR DESIGN}

The actuator was designed to achieve high displacements at low voltages. The mask layout of the actuator is shown in Fig. 1. The device is released in HF solution and is shown in Fig. 2. After the HF release process, the top electrode curves up due to the bending stress and difference in thermal expansion of the polysilicon and metal layers. When the voltage is applied on the contact pad, the electrostatic force between the curved cantilever and the bottom electrode on the substrate pulls the cantilever down. With a warped cantilever, the force closes the gap from the anchored end and gradually the zipping effect actuates the entire cantilever without increasing the biasing voltages.

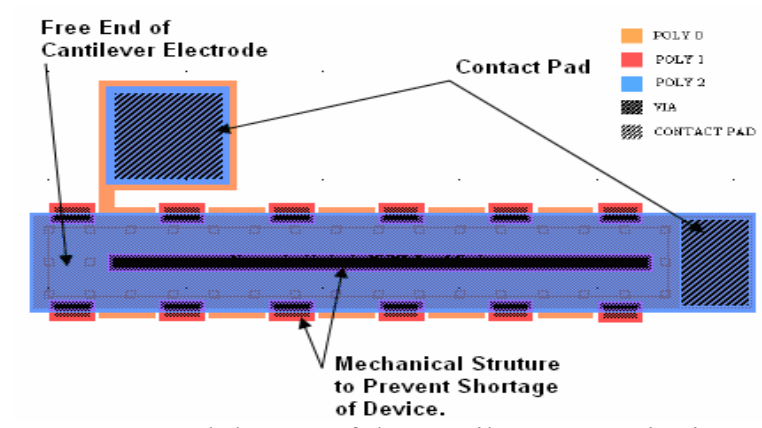

Figure 1 Mask layout of the cantilever type zipping actuator.

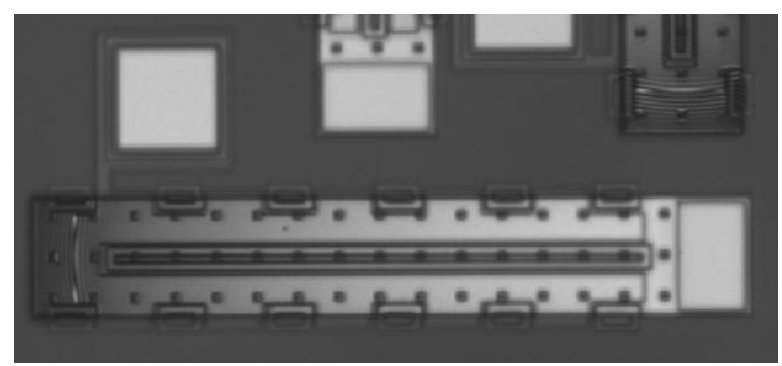

Figure 2 Fabricated zipping actuator after HF release.

\section{LUMPED MODELING ANALYSIS}

The lumped modeling analysis and the behavior of the actuator are presented in this section including various aspects such as stress induced bending of the cantilever beam, electrostatic force and the pull-in voltage of the actuator.

\subsection{Stress induced bending}

Micromachined cantilever beams are subject to residual stresses due to the fabrication process $[9,10]$. These stresses cause the cantilever beam to curl upwards. During the HF release process, the chips are heated, and upon returning to the room temperature, the cantilevers curve up more than the high temperature stress-induced state. The schematic of the cantilever shown in Fig. 3 has thickness of $h_{1}$ and $h_{2}$, residual stress of $\sigma_{1}$ and $\sigma_{2}$, and Young's modulus of $E_{l}$ and $E_{2}$ for polysilicon and gold layers, respectively. The force and moments acting on the structure are also shown in the figure [11]. The radius of curvature of the cantilever solely due to the residual stress is given by

$$
\rho=\frac{\left(w_{1} E_{1} h_{1}^{2}\right)^{2}+\left(w_{2} E_{2} h_{2}^{2}\right)^{2}+2 w_{1} w_{2} E_{1} E_{2} h_{1} h_{2}\left(2 h_{1}^{2}+3 h_{1} h_{2}+2 h_{2}^{2}\right)}{6 w_{1} w_{2} E_{1} E_{2} h_{1} h_{2}\left(h_{1}+h_{2}\right)\left(\frac{\sigma_{2}}{E_{2}}-\frac{\sigma_{1}}{E_{1}}\right)}
$$

And the total deflection at the tip of free end of cantilever is estimated as

$$
d=\rho\left(1-\cos \left(\frac{L}{\rho}\right)\right)
$$

where $L$ is length of the cantilever beam. 


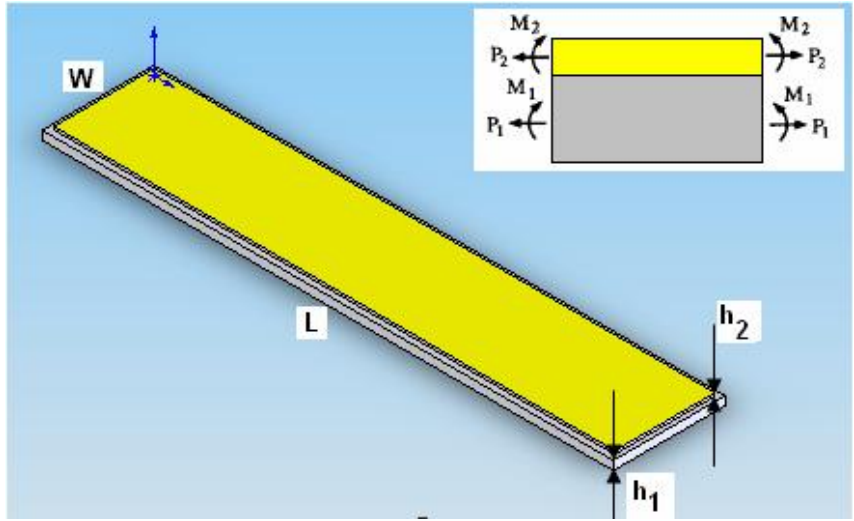

Figure 3 Schematic of the cantilever.

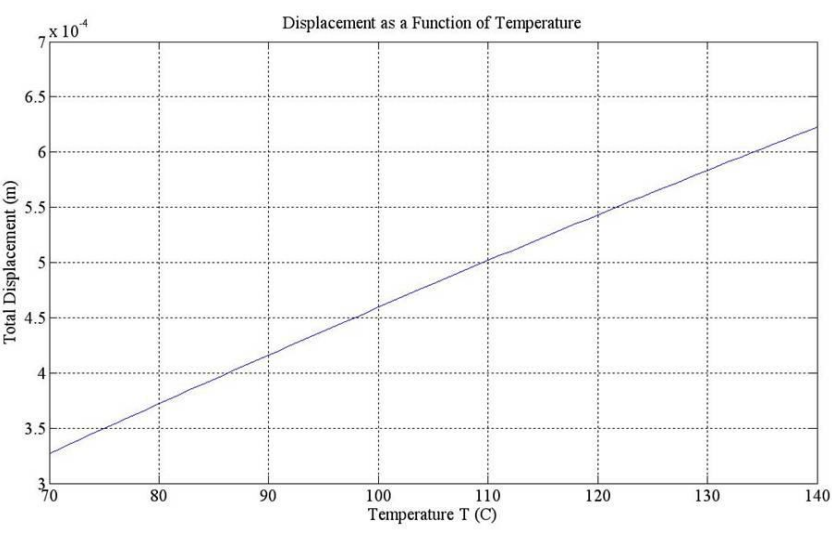

Figure 4 Displacement of cantilever as a function of temperature.

The radius of curvature due to the residual stress and change in temperature $\Delta \mathrm{T}$ is given by

$$
\rho=\frac{\left(w_{1} E_{1} h_{1}{ }^{2}\right)^{2}+\left(w_{2} E_{2} h_{2}{ }^{2}\right)^{2}+2 w_{1} w_{2} E_{1} E_{2} h_{1} h_{2}\left(2 h_{1}{ }^{2}+3 h_{1} h_{2}+2 h_{2}{ }^{2}\right)}{6 w_{1} w_{2} E_{1} E_{2} h_{1} h_{2}\left(h_{1}+h_{2}\right)\left(\alpha_{2}-\alpha_{1}\right) \Delta T}
$$

where $w_{1}$ and $w_{2}$ are the width, $\alpha_{1}$ and $\alpha_{2}$ are thermal expansion coefficients of polysilicon and gold layers, respectively.

The stress induced deflection, the deflection due to the residual stress and change in temperature, and hence the total deflection of the cantilever can be calculated. For a cantilever electrode that is $475 \mu \mathrm{m}$ long and $90 \mu \mathrm{m}$ wide, the total deflection solely due to stress is $16.285 \mu \mathrm{m}$. In our calculations, the induced stress due to fabrication processes is taken from the PolyMUMPs database as $7.8 \mathrm{MPa}$ for polysilicon and $23.3 \mathrm{MPa}$ for the gold layer. The total deflection for a cantilever electrode with a length of $475 \mu \mathrm{m}$ and a width of $90 \mu \mathrm{m}$ is $34.33 \mu \mathrm{m}$ at $100^{\circ} \mathrm{C}$ curing temperature. For a cantilever $1600 \mu \mathrm{m}$ long and $100 \mu \mathrm{m}$ wide, the deflection due to the residual stress and change in temperature is increased to $459.5 \mu \mathrm{m}$ at a curing temperature of $100^{\circ} \mathrm{C}$, as shown in Fig. 4.

\subsection{Electrostatic force}

A general analysis of the electrostatic force of the cantilever type zipper actuator can be performed by considering a parallel plate capacitor configuration composed of a moveable plate of a mass $M$ and a spring constant $K$ separated from the bottom electrode by an air gap $d_{o}$ [12]. The configuration is shown in Fig. 5. A constant voltage is applied between the top and bottom electrodes. Electrical energy is stored in the electric fields established by the charges accumulated in the capacitor due to the applied electric potential. The electrostatic force pulls the top electrode towards the bottom electrode causing the top electrode to deflect. This electrostatic force is inversely proportional to the air gap between the top and bottom electrodes. For lumped model analysis, damping within the system was neglected. The electrostatic force due to the stored charges is [13]

$$
F_{\text {electrostatic }}=\frac{\varepsilon_{0} A V^{2}}{2\left(d_{0}-z\right)^{2}}
$$

where $A$ is the area of moveable plate, $\varepsilon_{0}$ is the permittivity of free space, $d_{o}$ is the initial gap between the top and bottom electrodes, $z$ is the displacement, and $V$ is the applied potential. 


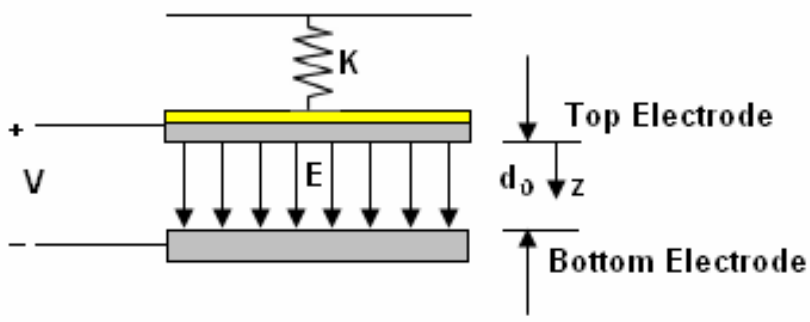

Figure 5 Parallel plate configuration of a zipper actuator.

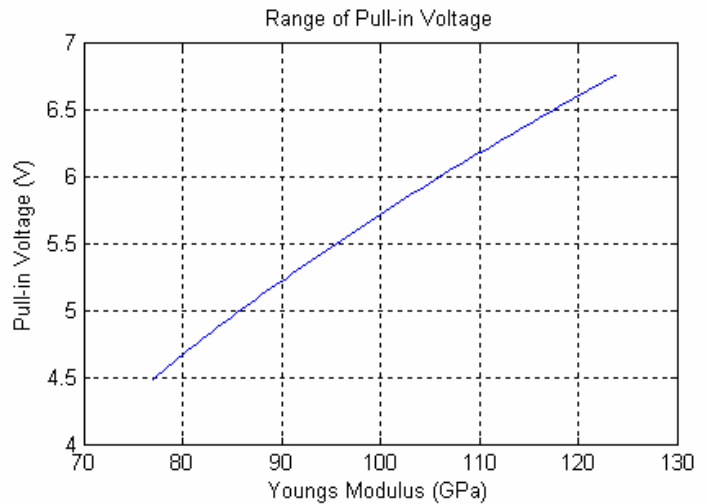

Figure 6 Range of the pull-in voltage.

\subsection{Pull-in voltage}

The pull-in action is related to the balance between elastic and electrostatic forces. When an electric potential is applied between the two electrodes, the electrostatic force generated causes the top electrode to collapse towards the bottom one. The elastic force is a linear function of the top electrode displacement and the electrostatic force is inversely proportional to the air gap between the two electrodes. When the applied voltage is increased, the top electrode slowly snaps towards the bottom electrode. At a certain input voltage, the electrostatic force becomes larger than the elastic force and the system cannot reach a force balance causing the top electrode to collapse onto the bottom electrode. This phenomenon is called pull-in and the voltage at this point is called the pull-in voltage. Chowdhury et al. [14] have formulated a closed-form expression for the pull-in voltage which is given by

$$
\begin{aligned}
& V_{P I}=\sqrt{\frac{2 \widetilde{E} h^{3} d_{0}}{8.37 \varepsilon_{0} l^{4}\left(\frac{5}{6 d_{0}{ }^{2}}+\frac{0.19}{d_{0}{ }^{1.25} w^{0.75}}+\frac{0.19}{d_{0}{ }^{1.25} l^{0.75}}+\frac{0.4 h^{0.5}}{d_{0}{ }^{1.5} w}\right)}} \\
& \widetilde{E}=\frac{E}{1-v^{2}}
\end{aligned}
$$

where $E$ is the Young's modulus and $v$ is the Poisson ratio.

This formulation assumes that the top electrode has only one layer. The top electrode of a zipper actuator in this paper has two layers: a $0.5-\mu \mathrm{m}$ thick gold layer deposited on top of a polysilicon layer. The nominal Young's modulus of polysilicon is $168 \mathrm{GPa}$ and that of gold is $77.2 \mathrm{GPa}$ [15]. Assumption is made that if the polysilicon layer is thin, the Young's modulus of gold should also contribute to the total Young's modulus. We assumed that the Young's modulus of the bi-layer cantilever is between $77.2 \mathrm{GPa}$ and $168 \mathrm{GPa}$. The range of the pull-in voltage for the cantilever actuator with a length of $475 \mu \mathrm{m}$ and a width of $90 \mu \mathrm{m}$ is calculated and shown in Fig. 6. These values indicate the pull-in voltage should be in the range of $4.5 \mathrm{~V}$ to $6.7 \mathrm{~V}$.

\section{EXPERIMENTAL RESULTS}

Zipper actuators with various lengths and widths were fabricated using the PolyMUMPs process. The MUMPs chips were released using the HF release process and a sequential curing procedure. The scanning electron micrograph (SEM) images of the various configurations are shown in Fig. 7. 

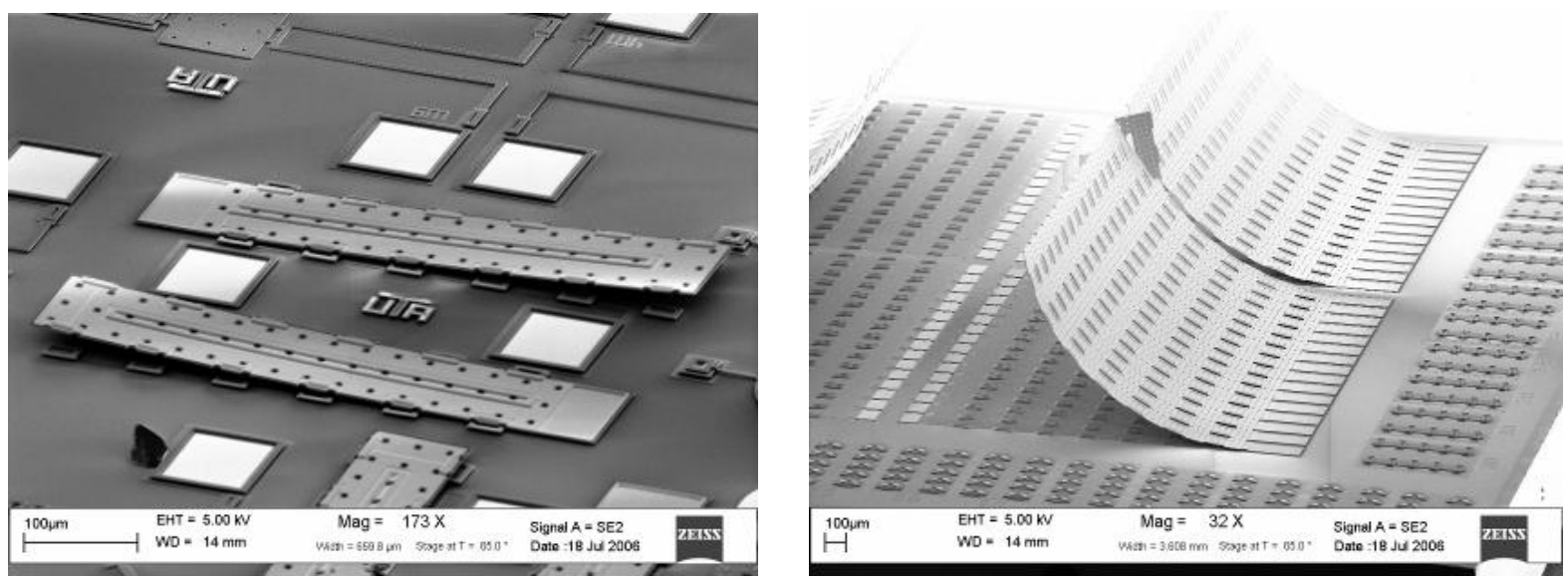

Figure 7 SEM images of various configurations of zipper actuators.

The maximum tip deflection of the actuator was determined experimentally using an optical profiler and a reflective microscope. The actuator tip deflections at various input voltages were first measured. The input voltage was varied from $0 \mathrm{~V}$ to $45 \mathrm{~V}$. Fig. 8 shows the plot of displacement as a function of biasing voltage for a zipping actuator with a length of $495 \mu \mathrm{m}$ and a width of $90 \mu \mathrm{m}$. For the analysis of pull-in voltages, the biasing voltage was varied from $0 \mathrm{~V}$ to $45 \mathrm{~V}$ in a step of $0.2 \mathrm{~V}$. The height of the cantilever decreases slowly until $7.8 \mathrm{~V}$. The cantilever electrode then collapses to the bottom electrode at $7.8 \mathrm{~V}$. The deflection of the electrode is well controlled with a voltage below $7.8 \mathrm{~V}$. At the pull-in voltage, the electrode collapses at a voltage increment of $0.05 \mathrm{~V}$. The pull-in voltage of $7.8 \mathrm{~V}$ includes the potential drops due to electrical resistances in the polysilicon biasing lines, the contact pads and the probe tips. This explains the higher pull-in voltage than the predicted one.

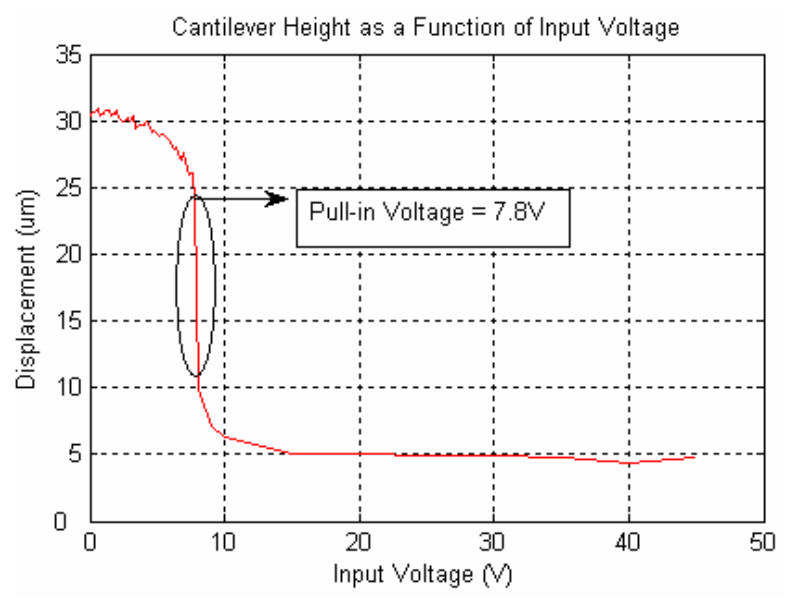

Figure 8 Deflection as a function of input voltage.

\subsection{Optical scanning}

The electrostatic zipping actuator has a top layer of gold. This highly reflective surface can be used to reflect a laser beam. The top electrode of the zipper actuator when actuated moves towards the bottom electrode. When the voltage is released, the electrode returns to its initial position. The arrangement to demonstrate the optical scanning is shown in Fig. 9 consisting of a voltage source, a microscope and a video camera setup to probe and monitor the actuation. The actuator is probed using $25-\mu \mathrm{m}$ nickel coated probe tips connected to magnetic manipulators as shown in Fig. 10. Fig. 11 shows an SEM photo of the assembled scanner. The $633-\mathrm{nm}$ He-Ne laser beam is carried by a single- mode optical fiber with a diameter of $125 \mu \mathrm{m}$ to the zipping actuator. The distance between the end of the fiber to the reflection spot 


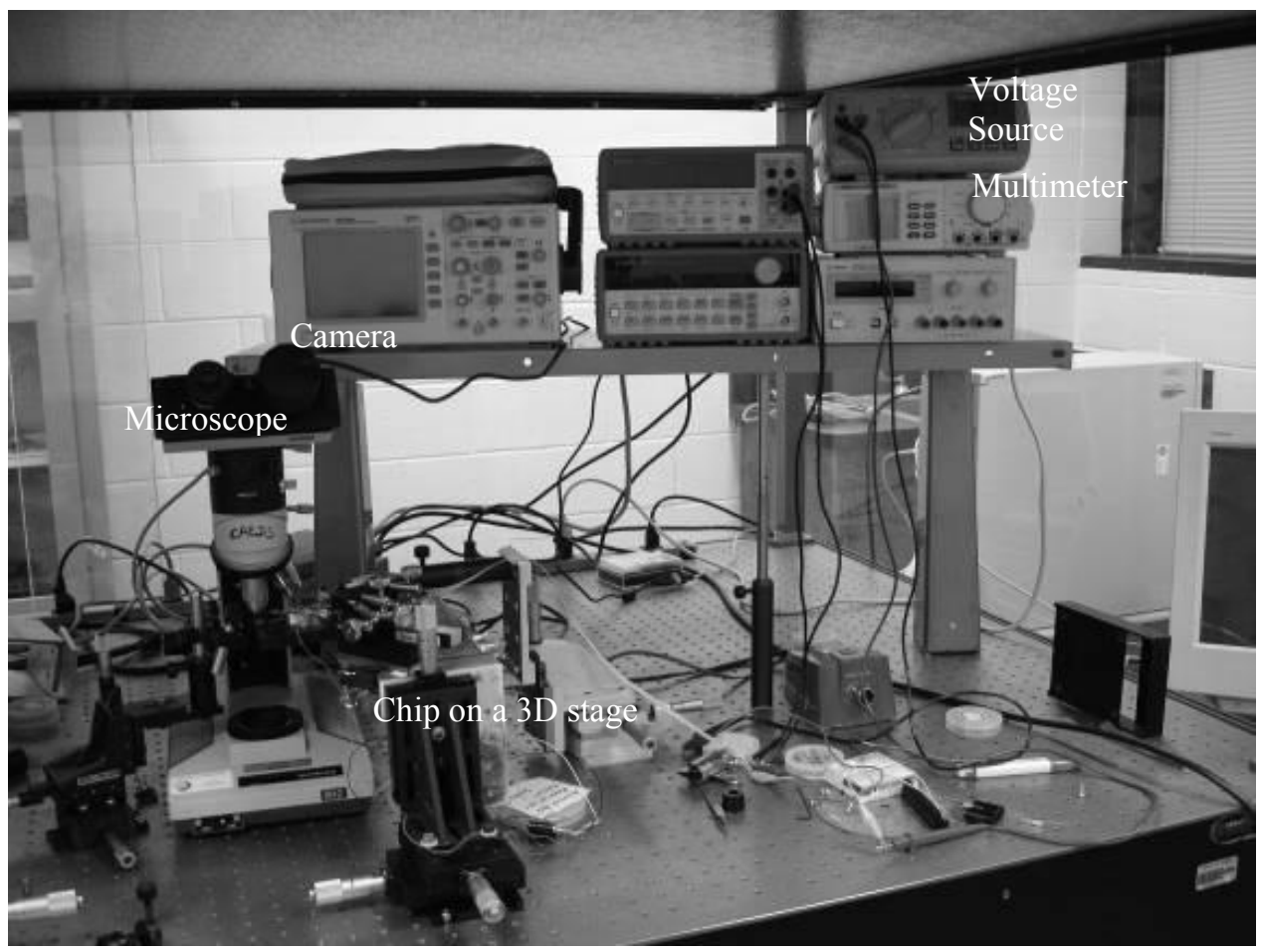

Figure 9 Setup for optical scanning using the zipping actuator.

The $1600-\mu \mathrm{m}$ long actuator is probed with biasing voltages in the range of $10-50 \mathrm{~V}$ and the motion of the reflected beam spot is traced. The beam is found to trace a path from a position vertically above the electrode, when not actuated, to a point nearly in-line with the fiber and lower electrode when a biasing voltage of $24 \mathrm{~V}$ is applied, as shown in Fig. 12. The biasing voltage is chosen to be much above the pull-in voltage to ensure maximum deflection. The scanning angle is estimated to be around $70^{\circ}$.

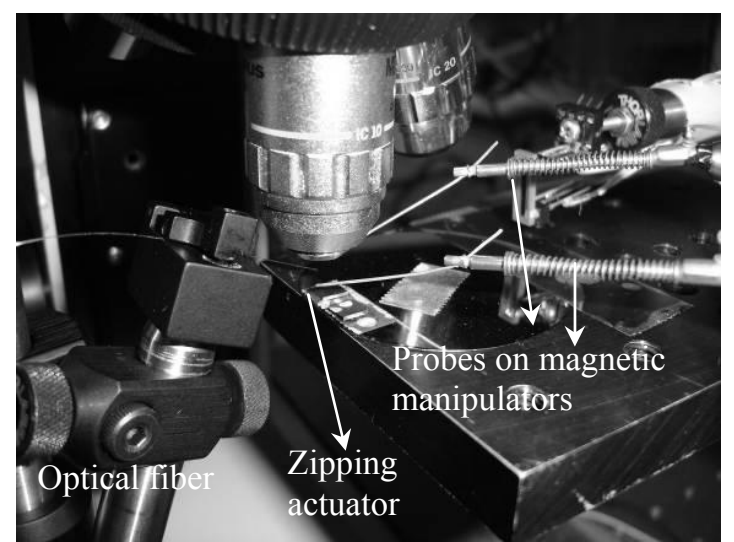

Figure 10 Probing arrangement.

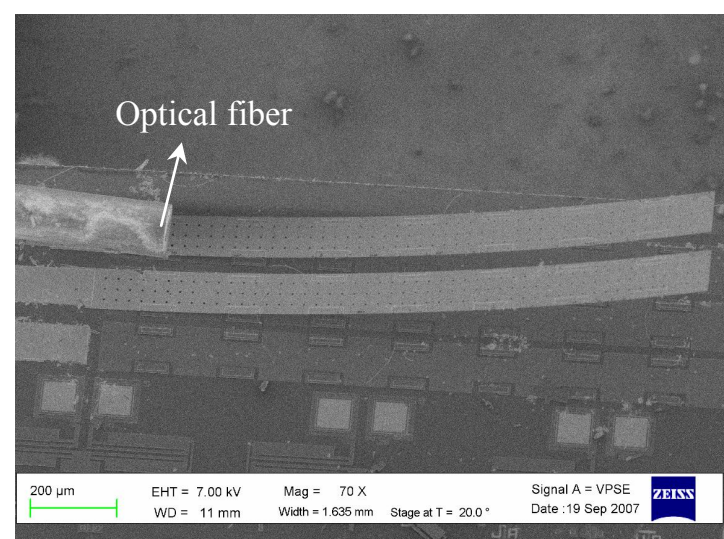

Figure 11 SEM image of the zipper actuator and fiber assembly. 


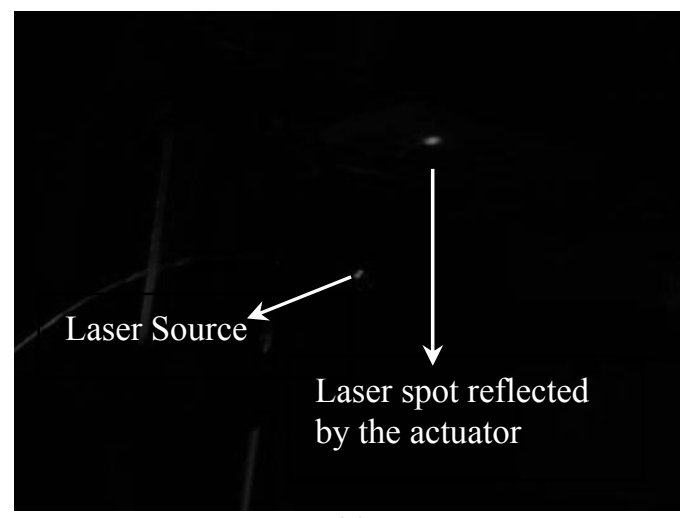

(a)

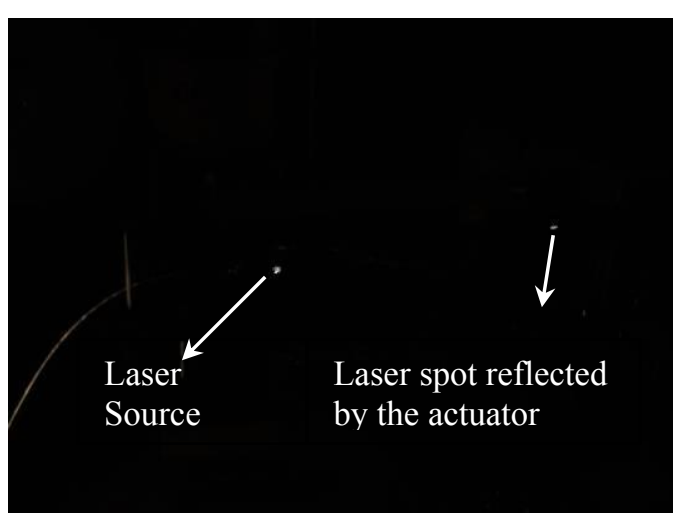

(b)

Figure 12 Laser reflection (a) without a biasing voltage and (b) with the maximum biasing voltage.

\section{CONCLUSIONS}

In this paper, a cantilever-type electrostatic zipper actuator is presented. The device is fabricated using multilayer polysilicon process, PolyMUMPs. The actuator operates at low voltages without the requirement of a dielectric isolation layer. The actuator is characterized in terms of electrostatic forces, pull-in voltages, and deflections. A mechanical structure is designed to prevent the electrical shortage of the device without the use of dielectric material. The theoretical and experimental results are verified. The ability of the zipper actuator to function as an optical scanner is shown experimentally. The scanning actuator can be utilized in general imaging or scanning applications.

\section{ACKNOWLEDGEMENT}

The authors would like to express their gratitude to Dr. Nasir Basit and Mr. Dennis Bueno for their cleanroom assistance.

\section{REFERENCES}

[1]. J. Li, M. P. Brenner, J. H. Lang, A. H. Slocum, and R. Struempler, "DRIE-Fabricated curved electrode zipping actuators with low pull in voltage", TRANSDUCERS 03, The $12^{\text {th }}$ International Conference on Solid State Sensors, Actuators and Microsystems, Boston, 2003.

[2]. M. Shikida, K. Sato, and T. Harada, "Micromachined S-shaped actuator," IEEE Sixth International Symposium on Micro Machine and Human Science, 8:167-172, 1995.

[3]. M. Yamaguchi, S. Kawamura, K. Minami, and M. Esashi, "Distributed electrostatic micro actuator", IEEE Conference on Microelectromechanical Systems, FL, 18-23, 1993.

[4]. R. Legtenberg, J. Gilbert, and S. D. Senturia, "Electrostatic curved electrode actuators", Journal of Microelectromechanical Systems, 6:257-265, 1997.

[5]. R. Jebens, W. Trimmer, and J. Walker, "Microactuators for aligning optical fibers", Sensors and Actuators, 20:65-73, 1989.

[6]. J. Branebjerg and P. Gravesen, "A new electrostatic actuator providing improved stroke length and force," IEEE Conference on Microelectromechanical Systems, Travemunde, Germany, 6-11, 1992.

[7]. N. Dhaubanjar "The design and analysis of optical scanners for optical coherence tomography," Master's Thesis, The University of Texas at Arlington, 2006. 
[8]. N. Dhaubanjar, S. M. N. Rao, Y. Cai, D. Popa, M. Chiao and J.-C. Chiao, "A cantilever-type electrostatic zipping actuator," SPIE International Smart Materials, Nano- \& Micro-Smart Systems Symposium, Smart Structures, Devices, and Systems Conference, Adelaide, Australia, Dec.10-13 2006.

[9]. V. K. Pamula, A. Jog, and R. B. Fair, "Mechanical property measurement of thin-film gold using thermally actuated bimetallic cantilever beams", Nanotech, Vol. 1, Chapter 8, pp.410-413, 2001.

[10]. M. T. Hou and R. Chen, "Effect of width on the stress-induced bending of micromachined bilayer cantilevers", Journal of Micromechanics and Microengineering, 13:141-148, 2002.

[11]. M. W. Judy, Y. Cho, R. T. Howe, and A. P. Pisano, "Self-adjusting microactuators (SAMS)", Proc. IEEE MEMS (MEMS'91), 51-56, 1991.

[12]. W. H. Chut, M. Mehreganyt, and R. L. Mullen, "Analysis of tip deflection and force of a bimetallic cantilever microactuator", Journal of Micromechanics and Microengineering, 3:4-7, 1993.

[13]. E. Saucedo-Flores', R. Ruelas', M. Floresl, Y. Cai and J.-C. Chiao, "Dynamic behavior modeling of MEMS parallel plate capacitors," Position Location and Navigation Symposium, pp. 15-19, 2004 .

[14]. S. Chowdhury, M. Ahmadi, and W. C. Miller, "A closed-form model for the pull-in voltage of electrostatically actuated cantilever beam", Journal of Micromechanics and Microengineering, 15:756-763, 2005.

[15]. W. N. Sharpe, Jr., Bin Yuan, and R. L. Edwards, "A new technique for measuring the mechanical properties of thin films," Journal of Microelectromechanical Systems, Vol. 6, No. 3, 1997. 